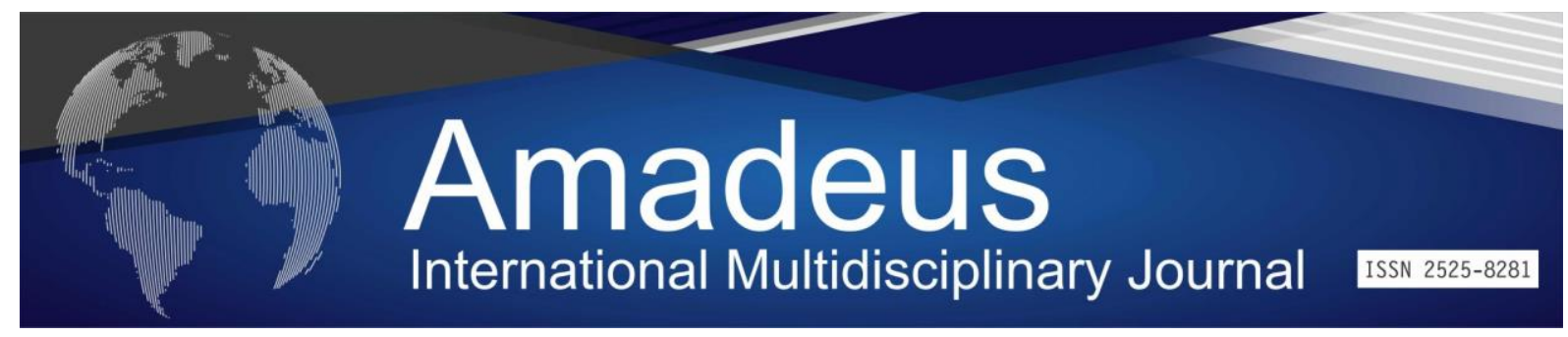

DOI: $10.14295 /$ aimj.v5i9.119

\title{
COVID-19 and Racial Issues: How this Disease has Generated Repercussions in African Americans
}

\author{
Modesto Leite Rolim Netol, \\ Camila Bezerra Nobre ${ }^{2}$, \\ Anne Karoline Avelino Silva ${ }^{3}$, \\ Camila Morais $\mathrm{Cruz}^{4}$, \\ Mabel Maria Sousa \\ Figueiredo ${ }^{5}$ \\ Taís Maia Landim
}

\begin{abstract}
Black people represent only 13\% of the US population, however they represent $30 \%$ of deaths caused by Covid-19. Many discrepancies like these are the result of structural inequalities that make black communities in the country more susceptible to contagion and more likely to develop severe forms of Covid-19, the disease caused by the new coronavirus. It is evident therefore, that as with any other social problem, the impacts caused by the new coronavirus are even more destructive in socially segregated niches. The crises in the health system and the economy that humanity has been through have opened up differences and intensely victimized the most vulnerable populations. African-Americans, the poor and individuals with low schooling are the people who suffer a potentially overwhelming effect, caused, to a great extent, by historical structural prejudice and by the stereotypes historically constructed by society.
\end{abstract}

Keywords: Covid-19; Racial issues; Social differences.

\footnotetext{
1 Pós-Doutor em Saúde Pública pela Universidade de São Paulo-USP e Professor do curso de Medicina da EstáciolFaculdade de Medicina de Juazeiro do Norte-FMJ; modestorolim@yahoo.com.br

${ }^{2}$ Doutora em Biotecnologia pela Universidade Estadual do Ceará-UECE e Professora da EstáciolFaculdade de Medicina de Juazeiro do Norte-FMJ; milinhanobre@gmail.com

${ }^{3}$ Estudante de medicina da Faculdade de Medicina de Juazeiro do Norte (Estácio-FMJ); anny_karoline12@ hotmail.com

${ }^{4}$ Estudante de medicina da Faculdade de Medicina de Juazeiro do Norte (Estácio-FMJ); camilamoraiscruz@gmail.com

${ }^{5}$ Estudante de medicina da Faculdade de Medicina de Juazeiro do Norte (Estácio-FMJ); mabelmariasousa@ gmail.com

${ }^{6}$ Estudante de medicina da Faculdade de Medicina de Juazeiro do Norte (Estácio-FMJ). tais2929@hotmail.com
} 


\section{COVID-19 e as Questões Raciais: Como essa Doença vem Gerando Repercussões em Afro-Americanos}

\begin{abstract}
Resumo: Os negros são apenas $13 \%$ da população dos EUA, todavia representam 30\% das mortes causadas por Covid-19. Muitas discrepâncias como essas são resultado de desigualdades estruturais que fazem com que comunidades negras no país fiquem mais suscetíveis ao contágio e tenham mais chances de desenvolver formas graves da Covid-19, a doença causada pelo novo coronavírus. Fica evidente, portanto, que assim como em qualquer outro problema social, os impactos causados pelo novo coronavírus são ainda mais destrutivos nos nichos socialmente segregados. As crises do sistema de saúde e da economia pelas quais a humanidade tem passado escancaram as diferenças e vitimizam intensamente as populações mais vulneráveis. Afro-americanos, pobres e indivíduos com baixa escolarização são as pessoas que sofrem um efeito potencialmente avassalador, causado, em muito, pelo preconceito estrutural histórico e pelos estereótipos construídos historicamente pela sociedade.
\end{abstract}

Palavras-chave: Covid-19; Questões raciais; Desigualdades sociais.

\section{Caro Editor,}

Os negros são apenas $13 \%$ da população dos EUA, todavia representam $30 \%$ das mortes causadas por Covid-19. As autoridades federais vincularam essas disparidades ao comportamento individual e solicitaram aos negros e outras comunidades de cor que "evitem álcool, tabaco e drogas" como se esse fosse um problema específico para esses grupos (The New York Times, 2020). As discrepâncias são resultado de desigualdades estruturais que fazem com que comunidades negras no país fiquem mais suscetíveis ao contágio e tenham mais chances de desenvolver formas graves da Covid-19, a doença causada pelo novo coronavírus (BBC, 2020). Parte do impacto desproporcional da pandemia do Covid-19 nas comunidades de cor tem sido por fatores estruturais que impedem essas comunidades de praticar o distanciamento social (Van Dorn, Cooney, Sabin, 2020).

Cerca de 90\% dos casos mais graves de Covid-19 envolvem pessoas com doenças crônicas, as quais são mais comuns e mais mortais em negros americanos (Garg et al, 2020). Há muito tempo que os americanos negros têm taxas mais altas de hipertensão, doenças cardíacas, diabetes e doenças pulmonares. Profissionais médicos disseram que o coronavírus 
agrava os desafios que surgem com essas doenças (The Washington Post, 2020a). Se os afroamericanos são mais propensos a sofrer as comorbidades que tornam o coronavírus mais mortal, é porque essas doenças estão ligadas à segregação e à pobreza concentrada que ainda marcam suas comunidades (The New York Times, 2020).

Essa realidade também está ligada à classe social, já que os afro-americanos têm maior probabilidade de terem empregos considerados essenciais durante a crise e, portanto, de se expor mais à infecção (Correio Braziliense, 2020). Os negros estão super-representados em comparação com a população geral da indústria de serviços de alimentação, hotelaria, taxistas e motoristas (The Washington Post, 2020a). Trabalhadores negros também são menos propensos a trabalhar em empregos que permitem o teletrabalho. Esse menor acesso ao teletrabalho é um lembrete de que os trabalhadores negros não são apenas vulneráveis aos impactos diretos à saúde do Covid-19, mas também têm maior probabilidade de serem vulneráveis às consequências econômicas dessa pandemia. Essa precipitação econômica também terá consequências para a saúde, talvez tão graves quanto a própria Covid-19 (The Guardian, 2020b).

A pandemia do COVID-19 levou à introdução de fortes medidas restritivas que estão tendo um efeito substancial na economia global, incluindo um aumento na taxa de desemprego em todo o mundo (Kawohl, W., Nordt C.,2020). A taxa de desemprego real dos afro-americanos pode ter atingido $19 \%$ em março. A razão pela qual os afro-americanos são os que mais sofrem com a crise é que, quando as decisões de demissão começam a ocorrer, os menos instruídos e aqueles com menos experiência tendem a ser liberados primeiro. Também existe discriminação contínua no local de trabalho (The Guardian, 2020a). Questões de desvantagem econômica se cruzam com questões de raça e etnia (Bavel et al ,2020)

As pessoas de cor são mais propensas a viver em áreas densamente compactadas e em situações habitacionais multigeracionais, que criam maior risco de propagação de doenças altamente contagiosas como a covid-19" (The Washington Post, 2020a). Ser pobre pode custar até 15 anos de expectativa de vida (The Guardian, 2020a). Mesmo quando as autoridades de saúde combaterem o coronavírus, os negros serão os últimos a se beneficiar, porque não terão acesso a testes rápidos e tratamentos avançados (CNN, 2020).

É preocupante que, nessa discussão sobre disparidades, algumas pessoas fiquem com a ideia equivocada de que há uma relação (biológica ou comportamental) entre ser negro e ser 
afetado (BBC, 2020). Informações falsas, memes e piadas surgem, dizendo que os negros são imunes a esse vírus, nas mídias sociais. Essas alegações infundadas sobre a resistência sobrenatural dos negros às doenças levaram a resultados de saúde devastadores ao longo dos anos, porque essas crenças afetam como elas são tratadas ou não. E mais alarmante: o combate ao covid-19 se tornará menos prioritário se os negros se tornarem o rosto do vírus? O vírus será armado contra as comunidades negras e piorará a hostilidade aberta do governo às pessoas de cor? (The Washington Post, 2020b). Emergências nacionais, pandemias, epidemias, o que eles fazem é destacar a desigualdade. O que vemos no Covid-19 não é diferente. Está destacando as disparidades raciais em todos os níveis que estão na nossa sociedade há muito tempo (The Guardian, 2020a).

Fica evidente, portanto, que assim como em qualquer outro problema social, os impactos causados pelo novo coronavírus são ainda mais destrutivos nos nichos socialmente segregados. As crises do sistema de saúde e da economia pelas quais a humanidade tem passado escancaram as diferenças e vitimizam intensamente as populações mais vulneráveis. Afro-americanos, pobres e indivíduos com baixa escolarização são as pessoas que sofrem um efeito potencialmente avassalador, causado, em muito, pelo preconceito estrutural histórico e pelos estereótipos construídos historicamente pela sociedade.

\section{Referências}

BBC. (2020). Coronavírus: por que a população negra é desproporcionalmente afetada nos EUA? Disponível em https://www.bbc.com/portuguese/internacional-52267566

Garg S, Kim L, Whitaker M, et al. (2020). Hospitalization Rates and Characteristics of Patients Hospitalized with Laboratory-Confirmed Coronavirus Disease 2019 - COVIDNET, 14 States, March 1-30, 2020. MMWR Morb Mortal Wkly Rep 2020;69:458-464. Disponível em https://www.cdc.gov/mmwr/volumes/69/wr/mm6915e3.htm

Correio Braziliense. (2020). Dados sugerem que Covid-19 mata mais afro-americanos nos EUA. Disponível em https://www.correiobraziliense.com.br/app/noticia/mundo /2020/04/07/interna_mundo,843016/dados-sugerem-que-covid-19-mata-mais-afroamericanos-nos-eua.shtml

The New York Times (2020). Why Coronavirus Is Killing African-Americans More Than Others. Disponível em https://www.nytimes.com/2020/04/14/opinion/sunday/coronavirusracism-african-americans.html?searchResultPosition=4 
CNN. (2020). Black Americans are being hammered by a double pandemic. Disponível em https://edition.cnn.com/2020/04/12/health/black-americans-hiv-coronavirus-blake/index.html

The Guardian (2020a). African Americans bear the brunt of Covid-19's economic impact. Disponível em . https://amp.theguardian.com/us-news/2020/apr/28/african-americansunemployment-covid-19-economic-impact

The Guardian (2020b). The coronavirus burden is falling heavily on black Americans. Why? Disponível em https://www.theguardian.com/commentisfree/2020/apr/16/black-workerscoronavirus-covid-19

Kawohl, W., Nordt , C. (2020). COVID-19, unemployment, and suicide, The Lancet. Psychiatry, The Lancet, v. 7, n.1, p. 389-390, may 2020 .Disponível em https://www.thelancet.com/pdfs/journals/lanpsy/PIIS2215-0366(20)30141-3.pdf

Bavel et al. (2020). Using social and behavioural science to support COVID-19 pandemic response, Nature Human Behaviour, Nature, v. 4, n.59, p. 463-471, may 2020. Disponível em https://www.nature.com/articles/s41562-020-0884-z.pdf

Van Dorn, Aaron; Cooney, Rebecca e; Sabin, Miriam L. (2020). COVID-19 exacerbating inequalities in the US. The Lancet World Report, v. 395, n. 10232, p. 1243-1244, abr. 2020. Elsevier BV. Disponível em https://www.thelancet.com/pdfs/journals/lancet/PIIS01406736(20)30893-X.pdf

The Washinton Post $\left(2020^{\mathrm{a}}\right)$. 4 reasons coronavirus is hitting black communities so hard. Disponível em : https://www.washingtonpost.com/politics/2020/04/10/4-reasons-coronavirusis-hitting-black-communities-so-hard/

The Wahington Post (2020b). The pathology of American racism is making the pathology of the coronavirus worse. Disponível em https://www.washingtonpost.com/outlook/ 2020/04/11/coronavirus-black-america-racism/

\section{How to cite this article (APA format):}

Rolim Neto, Modesto Leite; Nobre, Camila Bezerra; Silva, Anne Karoline Avelino; Cruz, Camila Morais; Figueiredo, Mabel Maria Sousa; Landim, Taís Maia (2020). COVID-19 and Racial Issues: How this Disease has Generated Repercussions in African Americans. Am. In. Mult. J., Jul to Oct. (9) 5, 18-22.

Received: 06/11/2020

Accepted: 07/07/2020 